\title{
Reliable Communication Systems for Monitoring of Transportation in Mining Industries
}

\author{
Muhammad Adib Kamali ${ }^{1}$, Khoirul Anwar ${ }^{2}$, Desti Madya Saputri ${ }^{3}$ \\ \{adibkamali@student.telkomuniversity.ac.id, anwarkhoirul@telkomuniversity.ac.id, \\ destimadyasaputri@telkomuniversity.ac.id \} \\ Center for Advanced Wireless Technologies (AdWiTech), \\ School of Electrical Engineering, Telkom University
}

\begin{abstract}
The transportation route in mining areas consists of various complex fields, where reliable communication systems called mining communication system to monitor the transportation is important and urgent. This paper designs a reliable communication system for monitoring transportation in the mining industry by using a channel model that is suitable for mining environmental conditions. The mining communication system consists of a Base Station (BS) and sensors on a mine train. This paper also evaluate the use of relays to obtain better performances. Channels for mining communication systems are predicted using the Statistical Spatial Channel Model (SSCM) concept for mining areas in Indonesia. Furthermore, channel capacity on each link between S-BS, S-R, and R-BS are obtained and calculate outage probability as the theoretical basis for mining communication systems. We found that relay helps significantly the improvement on biterror-rate (BER) and frame-error-rate (FER) perfomances.
\end{abstract}

Keywords: Mining communication system, relay, channel model, Repetition codes.

\section{Introduction}

The transportation route in mining areas consists of various complex fields, where reliable communication systems called mining communication system to monitor the transportation is important and urgent. This paper consider a mining communication system that consists of a Base Station (BS), sensors (S) on a mine train. Relay (R) is evaluated to obtain better performances. Furthermore, channel capacity on each link between S-BS, S-R, and R-BS are obtained and calculated based on outage probability as the theoretical basis for mining communication systems. The outage probability representing the theoretical performances of mining communication systems. It can be observed by the estimate of channel capacities S-BS, $\mathrm{S}-\mathrm{R}$, and R-BS where is obtained from the power delay profile (PDP) of mining area.

Beside that a reliable mining communication system can be achieved by using a channel model that is suitable for mining environmental conditions becuase it has been used effectively in performance evaluation, for example, in [1] based on real field experiment in Germany. The Grasberg mine owned by PT Freeport Indonesia (PTFI) where is the largest gold mine and the second largest copper mine in the world is assumed as a representative channel of a mining communication. Channels that are suitable for mining communication systems is predicted using the Statistical Spatial Channel Model (SSCM) concept for The Grasberg mine

by New York University. NYUSIM use statistical spatial channel model (SSCM) technique to 


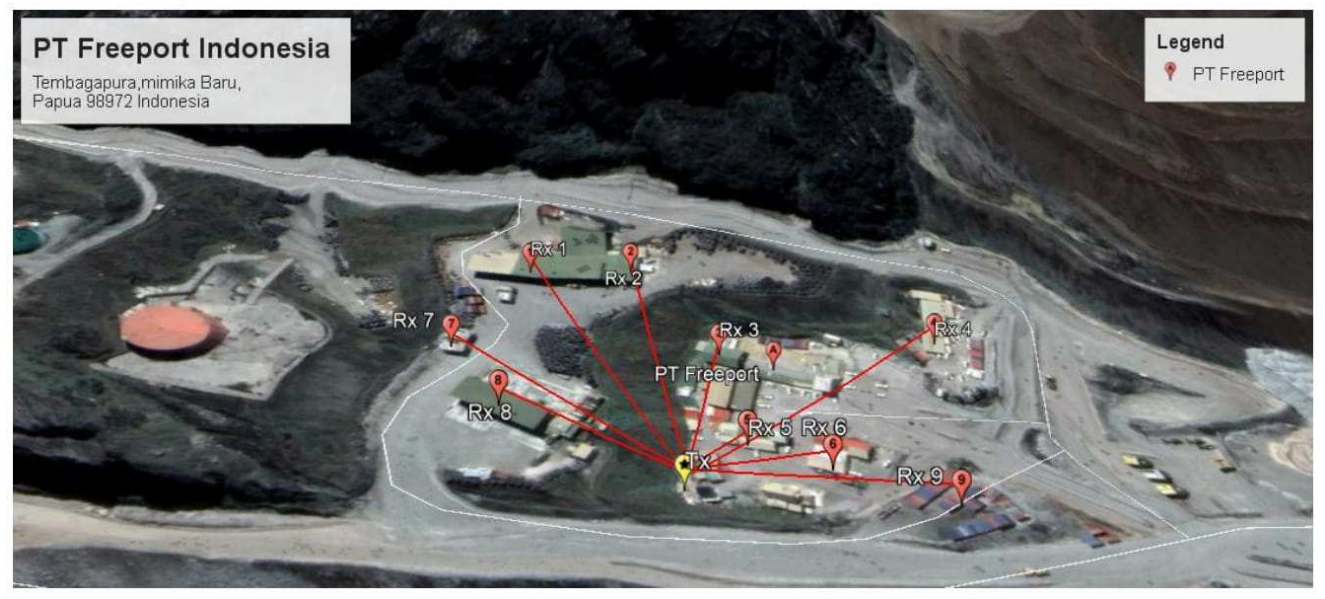

Figure 1. Grasberg mine open pit as channel modeling simulation area.

model the channel of mining communication systems. SSCM measures the channel with a lot of samples and analyze statistically by taking into account the spatial characteristic of the environment conditions including types of antenna [2].

Furthermore, channels where are obtained by NYUSIM evaluated with Repetition codes with coding rate $\left(R=\frac{1}{3}, \frac{1}{5}\right)$. Relay $\mathrm{R}$ also added up to mining communication system to get improvment of the performances.

The results of the computer simulations confirm that improvement on bit error rate (BER) performances from Relay $\mathrm{R}$ significantly outperforms Repetition codes $\left(R=\frac{1}{3}, \frac{1}{5}\right)$. A combination of Relay $\mathrm{R}$ and Repetition codes has excellent performances.

Table 1. NYUSIM channel simulator parameter input.

\begin{tabular}{ll}
\hline Frequency & $915 \mathrm{MHz}$ \\
\hline RF Bandwidth & $20 \mathrm{MHz}$ \\
Scenario & Umi \\
Environment & NLOS \\
T-R Separation - Lower Bound & 200 \\
Upper Bound & 200 \\
TX Power & $30 \mathrm{dBm}$ \\
Number of RX & 1000 \\
Baromatric Pressure & 1012.25 \\
Humidity & $56 \%$ \\
Temperature & $16 \mathrm{C}$ \\
Rain rate & $113.9 \mathrm{~mm} / \mathrm{h}$ \\
Polarization & $\mathrm{Co}-\mathrm{Pol}$ \\
Foliage Loss & No \\
Follage Attenetion & No \\
\hline
\end{tabular}




\section{System Model}

This paper consider bandwidth $B$ of $10 \mathrm{MHz}$ for the frequency $F$ of $915 \mathrm{MHz}$ which is used in commercially available mining communication systems and tracking systems [3]. The $B$ and $F$ used to predict channels of mine areas and validating mining communication systems. Orthogonal frequency division multiplexing with cyclic prefix (CP-OFDM) added up because this paper using multipath carrier. In addition, this paper using $\mathrm{R}$ to achieve improvement performances [4], using Repetition codes for low power consumption.

\subsection{Channel Modeling}

The Grasberg mine climate can be used as a representation channel of mining communication preliminary study. Barometric pressure, humidity and temperature in The Grasberg mine are used as climate parameters input of NYUSIM channel simulator. This model represent a mining environment due to focus from this paper is the mining area with an open pit mine. A distance between the sensor S and the receiver BS set 200 meters. Parameters for channel modeling shown in Table 1. In this paper these parameters as an input of NYUSIM channel simulator to generate PDP, therefore, NYUSIM channel simulator produced instantaneous PDP that representative channel of mining areas. . PDP that obtained by NYUSIM used to calculate channel capacity on each link between S-BS, S-R, and R-BS.

\subsection{Channel Capacity}

The channel capacity of $C$ is the maximum value of mutual information of the total information sent and received, where the error probability approaches zero when condition coding rate $R$ in under $C$ [5]. Calculation of channel capacity is important in designing a mining communication system because when mine trains run, the channel capacity $C$ will change due to changes in position and changes in environmental conditions that are passed by the mine train. This channel capacity limit determines the maximum data speed data rates that can be achieved without the existence of delay or complexity of the interference of the encoder or decoder. Channel capacity is influenced by the width of the transmission bandwidth and PDP. The calculation of channel capacity is also based on the Shannon Capacity theorem as

$$
C=B \cdot \log _{2}(1+\gamma)
$$

where $C$ is channel capacity, $B$ is bandwidth in Hertz units, and $\gamma$ is a ratio of signal power to the noise power (SNR).

\subsection{Repetition Codes}

The basic concept of Repetition Codes is to repeat the message several times. Repetition Codes can cover up messages lost when passing through a noise channel. Repeating each bit three times allows to correct one error in each group of three bits, but not more error. Example of decoder of Repetition codes shown in Table 2. 
Table 2. Example of encoder repetition codes.

\begin{tabular}{ccc}
\hline Coding Rate $R$ & Message bit $b$ & Encoded data $r$ \\
\hline$\frac{1}{3}$ & 0 & 000 \\
$\frac{1}{5}$ & 1 & 111 \\
& 0 & 00000 \\
& 1 & 11111 \\
\hline
\end{tabular}

\subsection{Relay}

There are various kinds of relay technology, for example, amplify and forward (AF), the relay function only amplifies the received signal then forward it to BS. Technique with AF is very simple, but it has the disadvantage that noise is transmitted too. Better relay technology is decode-and-forward (DF), which is a relaying technique which decodes first before forwarding to the destination BS. This technique in [6] is proven to be able to improve diversity and close to the performance of the Shannon limit. Therefore, this paper considers DF relay to get better performances of mining communication.

The proposed mining communication system with relay is shown in Figure 2. This paper assumes a single-relay single-source system. The relay is assumed to operate in half-duplex mode, in which the relay receives and transmits signals during the Phase-1 and Phase-2, respectively. Given that the distance between the Sensors $\mathrm{S}$ and the destination $\mathrm{BS}$ is $d_{s d}=d$, $\mathrm{S}$ to relay $\mathrm{R} d_{s r}=\frac{1}{4} d$, and $\mathrm{R}$ to $\mathrm{BS} d_{r b}=\frac{3}{4} d$. The gains of the $\mathrm{S}$ to $\mathrm{R}$ and $\mathrm{R}$ to $\mathrm{BS}$ links, given by

$$
\begin{gathered}
\operatorname{gain}_{s r}=\left(\frac{d_{s b}}{d_{s r}}\right)^{p}, \\
\operatorname{gain}_{r b}=\left(\frac{d_{s b}}{d_{r b}}\right)^{p},
\end{gathered}
$$

where $p$ is path loss exponent, $p$ between $2 \leq p \leq 6$ [7]. In this thesis $p=3.52$ that defined as

$$
\begin{aligned}
& \gamma_{s r}=\gamma_{s b}+21.19 d B \\
& \gamma_{r b}=\gamma_{r b}+4.4 d B
\end{aligned}
$$

Therefore, the received signals at the $\mathrm{R}$ and at $\mathrm{BS}$ are expressed as

$$
\begin{aligned}
& y_{s b}=\sqrt{\gamma_{s b}} \cdot h_{s b} \cdot s+n_{s b}, \\
& y_{s r}=\sqrt{\gamma_{s r}} \cdot h_{s r} \cdot s+n_{s r}, \\
& y_{r b}=\sqrt{\gamma_{r b}} \cdot h_{r b} \cdot s_{r b}+n_{r b},
\end{aligned}
$$

where $s$ represent the symbol vectors transmitted from the $\mathrm{S}$ to $\mathrm{BS}$ and $\mathrm{R}$ whre are modulated by complex -binary-phase shift keying (C-BPSK) with block length $K . h_{s b}, h_{s r}$ and 
$h_{r b}$ are the frequency-flat block-Rayleigh fading channel gains, which are constant in one block. The noise vectors $n_{s b}, n_{s r}$, and $n_{r b}$ are zero-mean Additive white Gaussian noise (AWGN) vectors with unit variances for S-BS, S-R, and R-BS links, respectively.

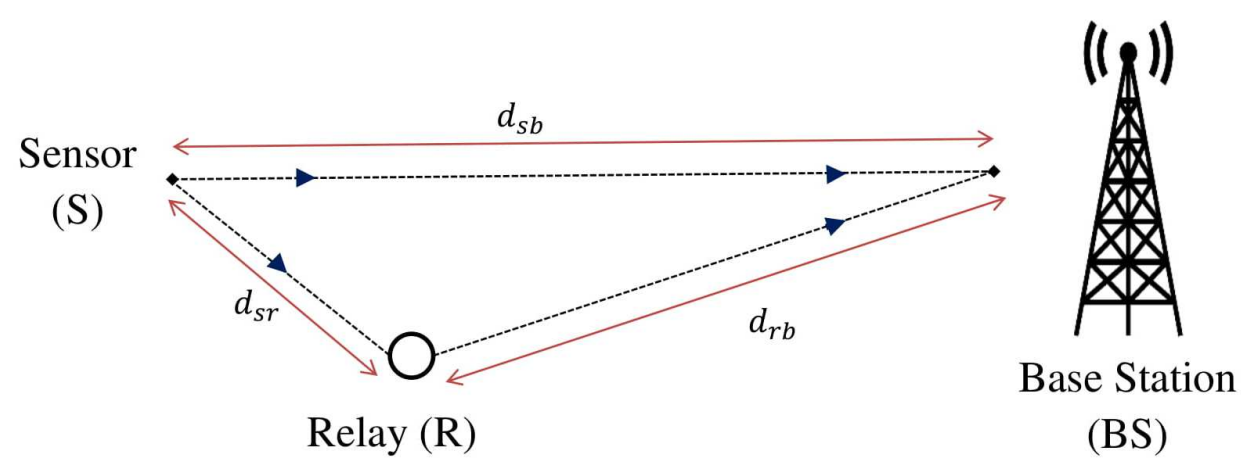

Figure 2. The communication system with relay

\section{Prosed Mining Communication System}

The proposed configuration of mining communication systems shown in Figure 3, where mine trains through the complex field. The proposed system model of the mining communication system is shown in Figure 4, where in phase-1 the sensors $\mathrm{S}$ broadcasts signal to the $\mathrm{R}$ and $\mathrm{BS}$ and in phase- $2 \mathrm{R}$ forward signal to the BS. At the transmitter $\mathrm{S}$, the binary information $b$ is encoded by Repetition codes Cs $\left(R=\frac{1}{3}, \frac{1}{5}\right), r$ modulated with C-BPSK Ms. Furthermore, $x$ are encoded into multiple carrier frequencies with inverse Fast Fourier Transform (IFFT) and added up Cyclic prefix Cp as OFDM scheme. In addition, this paper considers using OFDM with Minimum Mean Square Error (MMSE) equalization to compensate for the intersymbol interference (ISI). Symbol $x$ is then transmitted through the obtained mining area channel.

At the receiver side, the signals received from the sensors $\mathrm{S}$ during the Phase-1, denoted as $y_{s b}$. The signals received from the relay R during the Phase-2, denoted as $y_{r b}$. At the receiver $\mathrm{R}$, noise $n 2$ is added according to Gaussian distribution. At the receiver BS, there are two kinds of noise where noise $n 1$ is noise between S-BS link, and noise $n 3$ is noise between R-BS link. Noise $n 1, n 2$, and $n 3$ have different due to the different distances of S-BS, S-R, and R-BS that affected $\gamma$. In this paper noise AWGN $n$ are obtained by 


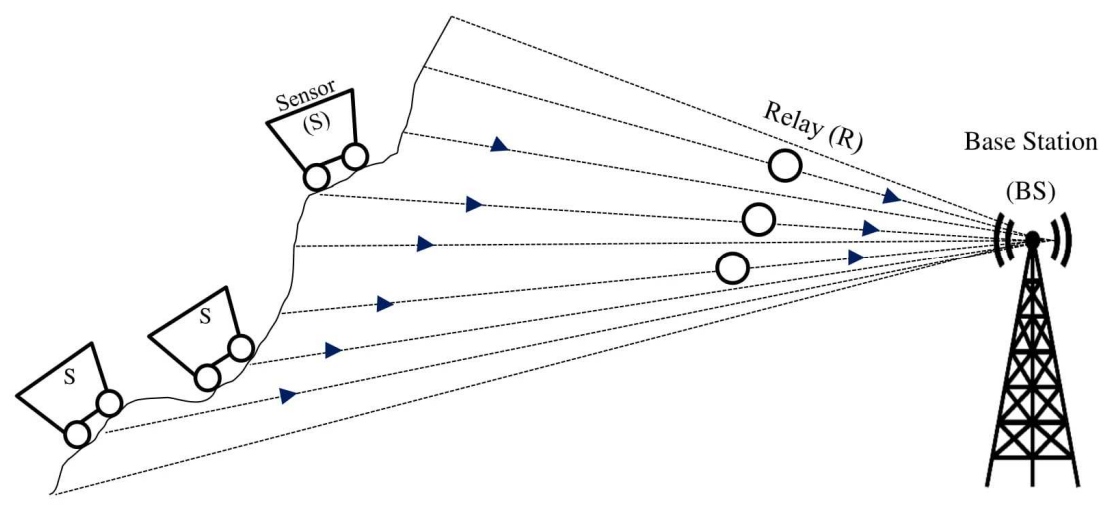

Figure 3. Configuration of the mining communication system.

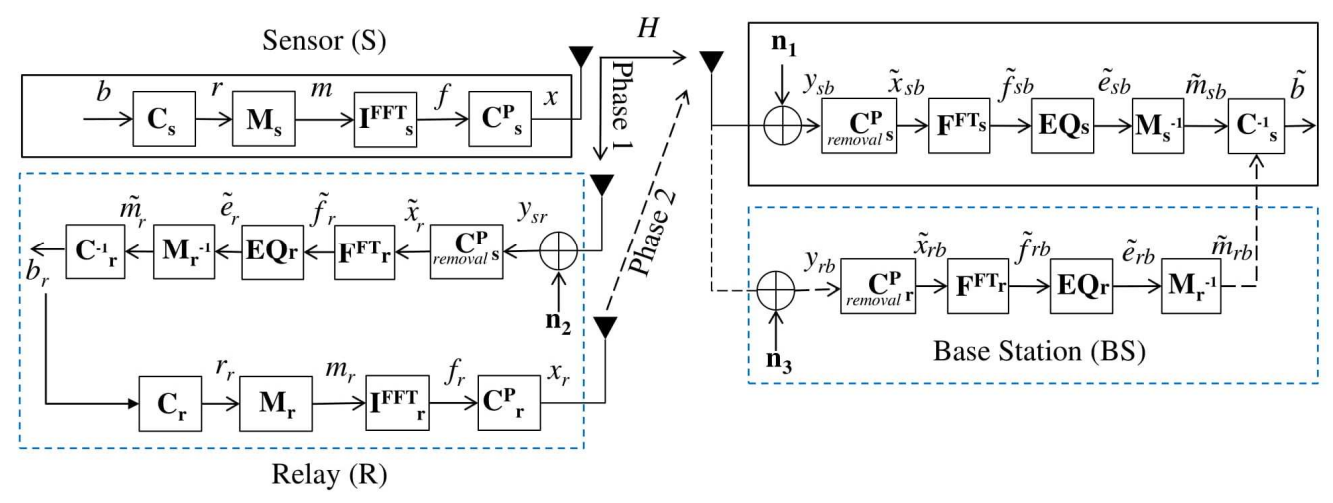

Figure 4. System model mining communication system.

$$
\mathrm{n}=\sigma .(\operatorname{randn}(1, \mathrm{~N})+\mathrm{j} *(\operatorname{randn}(1, \mathrm{~N}))
$$

where randn is a function of MATLAB to generate a random number with a normal distribution. Vector noise $n$ has Gaussian distribution with a number of $\mathrm{N}, \sigma$ is expressed as

$$
\sigma=\sqrt{ }(1 /(2 \cdot \gamma)
$$

$\mathrm{CP}$ removal module removes the CP to obtain $\tilde{x}$. Fast Fourier transform (FFT) module implement prior to equalization module EQ. We then perform demodulation $M^{-1}$ of C-BPSK.

At the relay, information bits $b_{s}$ are decoded similar process at BS. Repetition decoder is then performed on the output of $C_{r}^{-1}$ to obtain the binary source information $b_{r}$. The binary sequence $b_{r}$ are decoded into $x_{r}$ with similar process at $\mathrm{S}$. After that $x_{r}$ forwarded to the destination BS in Phase-2. The receiver BS performs $y_{s b}, \tilde{x}_{s b}, \tilde{f}_{s b}, \tilde{e}_{s b}, \tilde{m}_{s b}$ for the signal 
reception from the source during the Phase- 1 , and $y_{r b}, \tilde{x}_{r b}, \tilde{f}_{r b}, \tilde{e}_{r b}, \widetilde{m}_{r b}$ for the signal received from the relay during the Phase-2. Therefore, binary decision is made on $C_{r}^{-1}$ from addition of $m_{s b}$ and $m_{r b}$ to obtain the estimate $\tilde{b}$ of the source information $b$.

\section{Performances Evaluation}

\subsection{Representative PDP}

In this paper NYUSIM channel simulator produced Instantaneous PDP with 1000 trials to obtain one representative PDP of mining area. in this paper, calculation of the representative PDP based on [8] that take the 90th Cumulative distribution function (CDF) percentile of each in instantaneous PDP as a representative PDP. In the receiving side, the path which has a difference in propagation delay of less than $1 / B$ where $B$ is bandwidth in Hertz seen as one component in the power delay profile. The adding up power level shows the level of aggregate power of the multipath. Therefore, this paper mapped PDP According [9]. Figure 7 shows original and mapped PDP where is A representative PDP of mining areas.

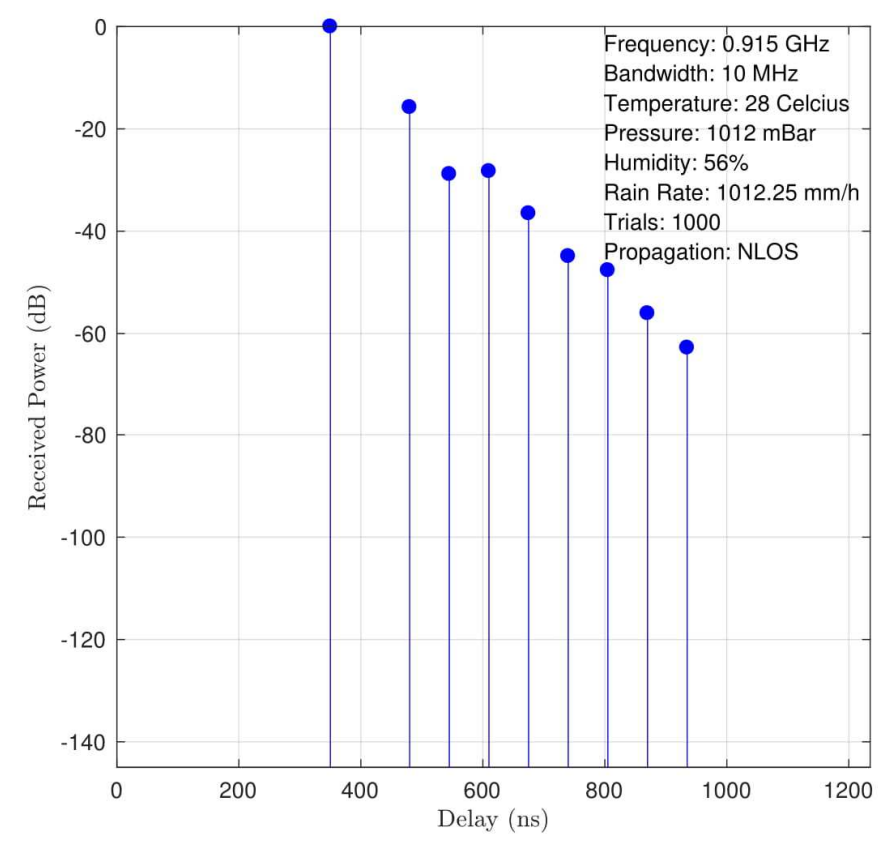

Figure 5. Original PDP 


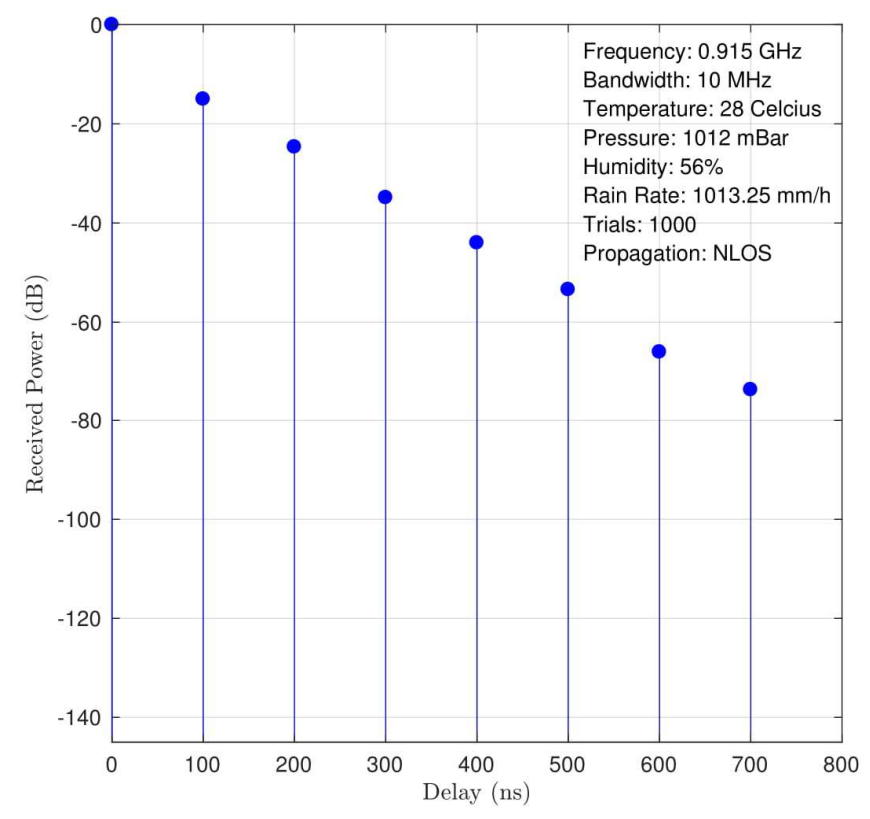

Figure 6. Mapped PDP A representative PDP of mining areas

\subsection{Outage Performances}

Outage Performances is the collection of outage probability that is needed in the design of a mining communication system to maximize the performance of a designed communication system. Outage Performances of mining area is shown in Figure 8. Based on curve outage performance that is obtained by MATLAB simulation, multipath has better performances than single path. High coding rate can improve outage performances of mining areas channel. This thesis consider using 8 path that obtained from channel modeling. In addition, information on outage performances is useful for improving power efficiency by regulating transmit power to follow the outage performances curve for each desired capacity and probability. Outage probability $P_{\text {otg }}$ is a probability of $P_{r}$ when the $C$ capacity drop below coding rate $R$ which results in information not being decoded correctly. Outage probability is calculated by

$$
P_{\text {otg }}=P_{r}(R>C)
$$

As long as $R \leq C$, the transmitted information can be decoded correctly on the receiver side [4]. PDP produced by NYUSIM Channel Simulator is used in calculating channels capacity of the mining communication system. The channel capacity $C$ in the multipath fading channel is expressed with

$$
C=\left(\frac{B}{L}\right) \cdot \sum_{n=1}^{L} \log _{2}\left(1+\left(\left|\psi_{n}\right|^{2} \cdot \gamma\right)\right)
$$


where $L$ is the FFT size. The $\psi_{n}$ symbol represents the eigen value of the multipath channel obtained from the result of representative PDP.

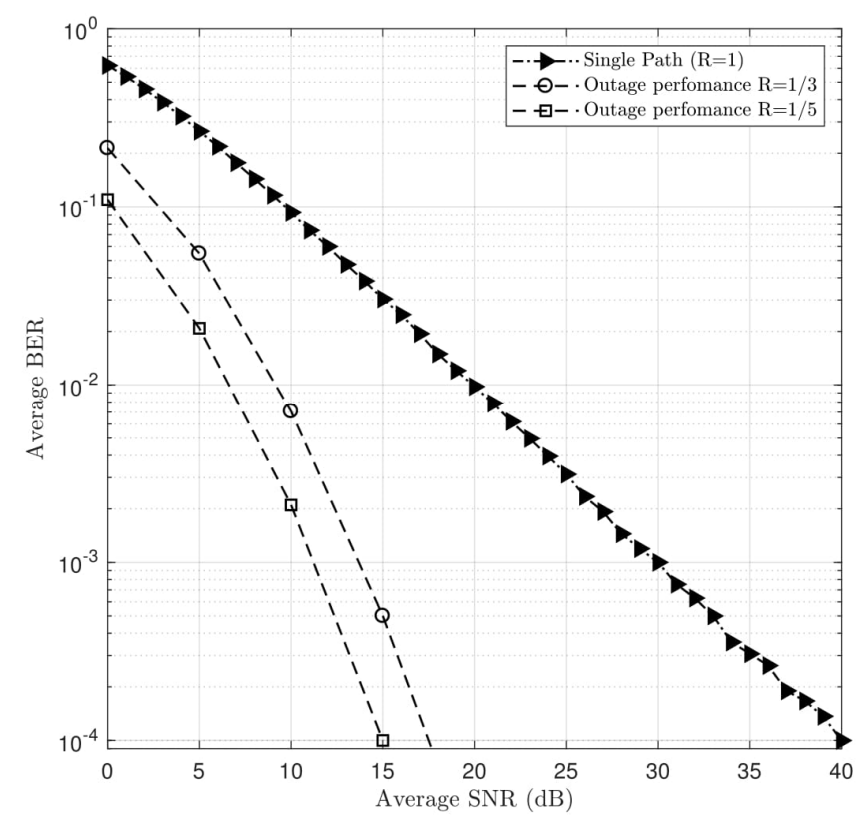

Figure 7. Outage Performances of mining areas

\subsection{Bit Error Rate}

This paper validates the proposed mining communication system by calculating Bit Error Rate (BER). Calculation BER in this paper used 200,000 experiments. This paper also evaluates BER performances by comparing BER performance on a channel Rayleigh fading with BER performance on a mining communication system. Performance on channel Rayleigh fading is the worst performance compared to performance on channel Rician Fading, and AWGN. Therefore, the performance evaluation in this paper is by comparing BER performances on a mining communication system with BER performances on Rayleigh fading. Calculation of BER on Rayleigh fading for modulation of C-BPSK with $\mathrm{R}=1$ is expressed as

$$
B E R_{\text {Rayleigh }}=\frac{1}{2}\left[1-\frac{1}{\sqrt{1+\frac{1}{\gamma}}}\right]
$$

where $\gamma$ is a ratio of signal power to the noise power (SNR). The performance of BER on the mining communication system is shown in Figure 9. Mining communication system without channel coding and relay achieve BER performance $10^{-4}$ at $\gamma=34.83 \mathrm{~dB}$. Mining 
communication system with Repetition codes $\left(R=\frac{1}{3}\right)$ achieve BER performance $10^{-4}$ at SNR $\gamma=24.73 \mathrm{~dB}$. Relay performances shows increases diversity when the SNR is large. Using relay achieve BER performance of $10^{-4}$ at SNR $\gamma=17.1 \mathrm{~dB}$.

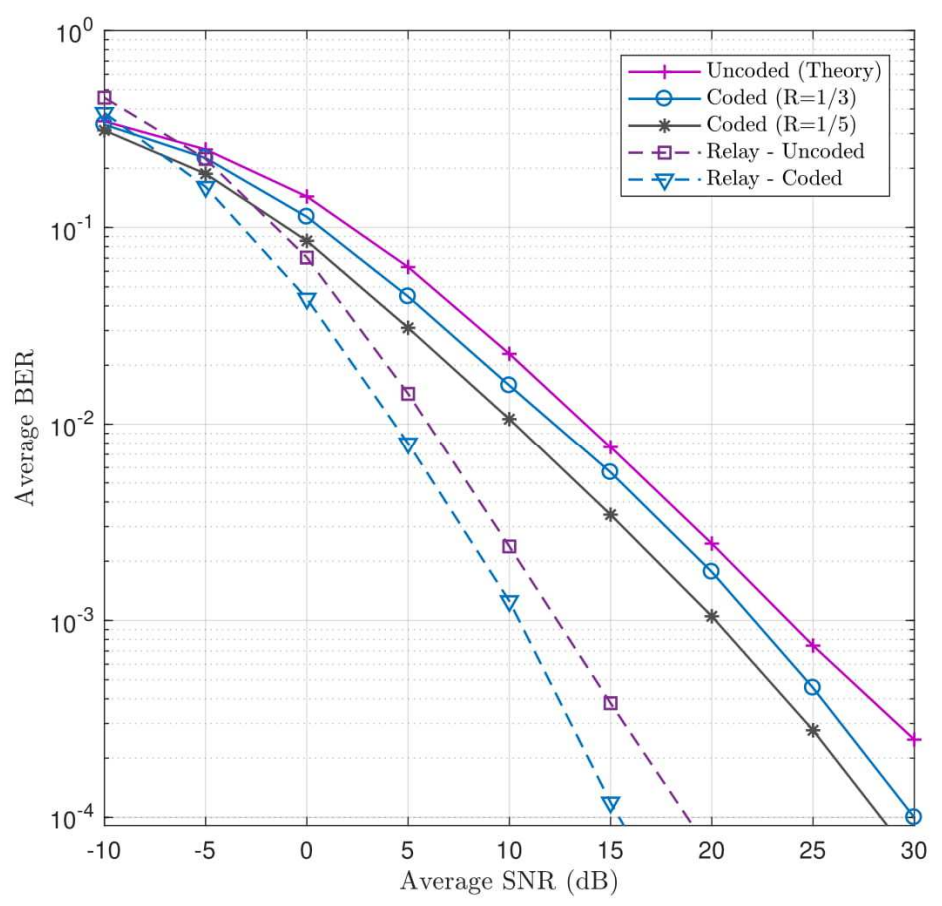

Figure 8. BER performances of mining communication system. 


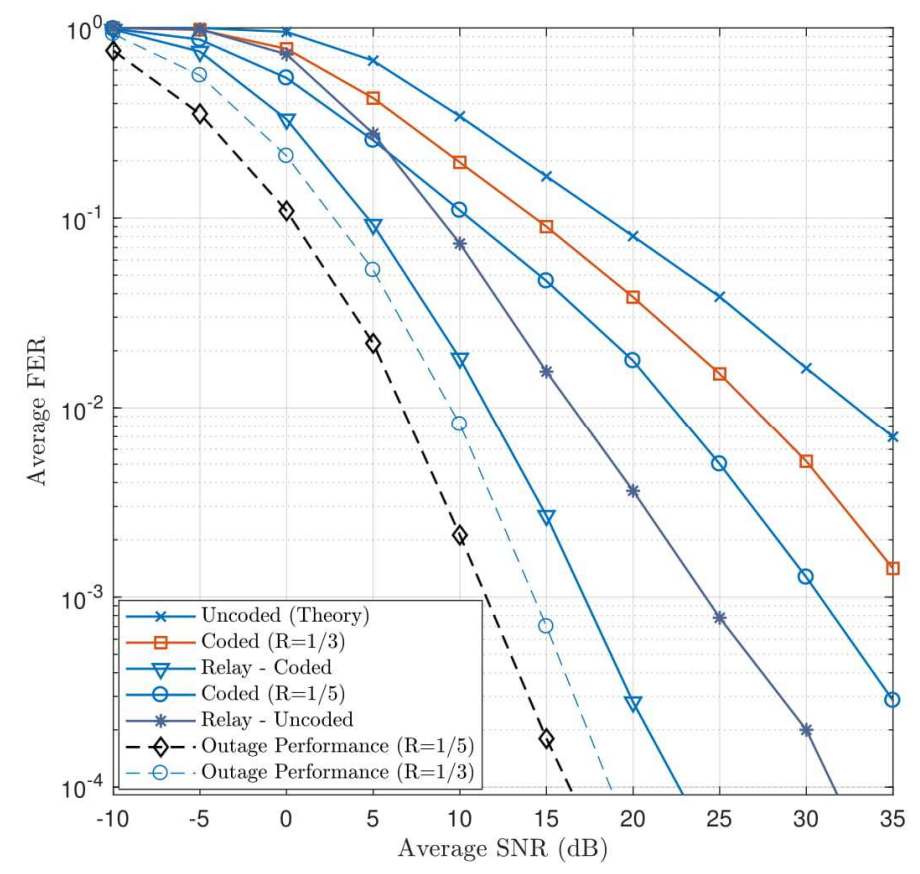

Figure 9. FER performances of mining communication system.

\subsection{Frame Error Rate}

This section provides FER performance results for all S-BS, S-R, R-BS links where are shown by Figure 10, where the outage performance curves for C-BPSK with coding rate $R=$ $\frac{1}{3}, \frac{1}{5}$ also shown for comparison. Outage probability $\left(R=\frac{1}{3}, \frac{1}{5}\right)$ of $10^{-4}$ is achieved at SNR $\gamma=$ $17.2,15.02 \mathrm{~dB}$. These curves are then used as the baseline comparison for practical FER performances. At FER of $10^{-4}$ the performance Relay-Uncoded is $\gamma=32.03 \mathrm{~dB}$ and the performance Relay-Coded is $\gamma=23.7 \mathrm{~dB}$. Mining communication system uncoded and with Repetition codes $\left(R=\frac{1}{3}, \frac{1}{5}\right)$ achieve FER performance $10^{-4}$ at SNR $\gamma>35 \mathrm{~dB}$.

\section{Conclusion}

This paper has proposed mining communication systems on the suitable channel with mining environment using NYUSIM channel simulator. This paper also has produced representative PDP of a mining area using a real field parameters of Grasberg mining area. In addition, the curve in BER performances show that increased power efficiency occurs when mining communication system used repetition codes and Relay. BER performance mining communication systems with Repetition codes can achieve better performances than uncoded. High coding rate help improving BER performances of mining communication systems. Relay performances shows increases diversity when the SNR is large since signal from $\mathrm{S}$ and signal 
from R added up at BS. The results of channel modeling in this paper are expected to be used as references in channel modeling experiments in the Indonesian mining area using devices in the field, such as channel sounder and Universal Software Radio Peripheral (USRP). In addition, the proposed mining communication system is expected to be used as a reference for the production of hardware and implementing a reliable communication system for monitoring mining industry transportation.

\section{References}

[1] K. Anwar and T. Matsumoto, "Field Measurement Data-based Performance Evaluation for Slepian-Wolf Relaying Systems," in IEICE General Conference, 2013.

[2] M. K. Samimi and T. S. Rappaport, "3-D Millimeter-Wave Statistical Channel Model for 5G Wireless System Design," IEEE Trans. Microw. Theory Tech., vol. 64, no. 7, pp. 2207-2225, Jul. 2016, doi: 10.1109/TMTT.2016.2574851.

[3] C. Zhou, T. Plass, R. Jacksha, and J. A. Waynert, "RF propagation in mines and tunnels: Extensive measurements for vertically, horizontally, and cross-polarized signals in mines and tunnels.," IEEE Antennas Propag. Mag., vol. 57, no. 4, pp. 88-102, Aug. 2015, doi: 10.1109/MAP.2015.2453881.

[4] M. Cheng, K. Anwar, and T. Matsumoto, "Outage probability of a relay strategy allowing intra-link errors utilizing Slepian-Wolf theorem," EURASIP J. Adv. Signal Process., vol. 2013, no. 1, 2013, doi: 10.1186/1687-6180-2013-34.

[5] A. Goldsmith, Wireless Communications. Cambridge University Press, 2005.

[6] K. Anwar and T. Matsumoto, "Spatially concatenated codes with turbo equalization for correlated sources," IEEE Trans. Signal Process., vol. 60, no. 10, pp. 5572-5577, 2012, doi: 10.1109/TSP.2012.2204259.

[7] R. Youssef and A. G. I. Amat, "Distributed serially concatenated codes for multi-source cooperative relay networks," IEEE Trans. Wirel. Commun., vol. 10, no. 1, pp. 253-263, Jan. 2011, doi: 10.1109/TWC.2010.102810.100422.

[8] E. Christy, R. P. Astuti, and K. Anwar, "Telkom University 5G Channel Models under Foliage Effect and Their Performance Evaluations," in Proceeding - 2018 International Conference on ICT for Rural Development: Rural Development through ICT: Concept, Design, and Implication, IC-ICTRuDEv 2018, 2018, pp. 29-34, doi: 10.1109/ICICTR.2018.8706848.

[9] Y.Xie, Z. Li, and M. Li, "Precise Power Delay Profiling with Commodity Wi-Fi," IEEE Trans. Mob. Comput., vol. 18, no. 6, pp. 1342-1355, Jun. 2019, doi: 10.1109/TMC.2018.2860991. 